\title{
An efficient algorithm for simulation of stochastic scattering cross-sections
}

\author{
$\begin{array}{ll}\text { M. Ganesh } & \\ & \text { S. C. Hawkins } \\ & \end{array}$
}

(Received 29 October 2012; revised 20 March 2013)

\begin{abstract}
We present an efficient algorithm for uncertainty quantification of scattering cross-sections of stochastic configurations comprising multiple two-dimensional sound-soft particles whose locations and orientations are random. Our algorithm has two components. Firstly, we use a high order generalised polynomial chaos approximation of the stochastic cross-sections in the random variables that requires realisation of the exterior sound propagation model for high order quadrature sampling parameters. For each such realisation, we use an efficient spectrally accurate algorithm for deterministic scattering simulations based on solving a boundary integral equation reformulation of the acoustic model. We present numerical results for a stochastic configuration of particles with rough surfaces. Our numerical result demonstrate the
\end{abstract}

http://journal.austms.org.au/ojs/index.php/ANZIAMJ/article/view/6300 gives this article, (C) Austral. Mathematical Soc. 2013. Published May 11, 2013, as part of the Proceedings of the 16th Biennial Computational Techniques and Applications Conference. ISSN 1446-8735. (Print two pages per sheet of paper.) Copies of this article must not be made otherwise available on the internet; instead link directly to this URL for this article. 
high order accuracy of both components of the algorithm for simulation of moments of the scattering cross-section of the configuration.

Subject class: $60 \mathrm{H} 35$

Keywords: acoustic scattering, stochastic, uncertainty quantification, generalized polynomial chaos

\section{Contents}

1 Introduction

2 Deterministic multiple scattering simulations

3 Uncertainty quantification

C127

4 Numerical results

C131

References

C135

\section{Introduction}

Quantifying scattering cross-sections of configurations measured from various directions plays a fundamental role in several applications which span over half a century $[2,8$, and references therein]. In particular, of recent interest is the use of such quantities in developing improved future climate models, reducing uncertainties in long term predictions [2, p. 57-58]. In such applications, because of the dynamic and uncertain nature of the configurations, the locations and orientations of particles in the associated computer models need to be taken as random variables. Consequently, parameters describing the stochastic configurations are elements in appropriate probability spaces. The direction dependent scattering cross-sections are random fields and their statistical moments (such as average scattering properties) are crucial tools 
for uncertainty quantification in several applications. While electromagnetic scattering by three dimensional configurations is of practical interest in climate applications, quantifying scattering cross-sections for the scalar twodimensional (acoustic or polarized electomagnetic) counterparts of the model is an important stepping stone for solving the full model problem.

In this article, we develop efficient tools for uncertainty quantification of the scattering cross-section of a stochastic sound-soft acoustic configuration $\mathrm{D}(\omega) \subset \mathbb{R}^{2}$ situated in a homogeneous medium. Here $\omega$ is an element of $\Omega$ where $(\Omega, \mathcal{F}, \mathbb{P})$ is a probability space and $\Omega$ is the set of possible outcomes, $\mathcal{F}$ is a $\sigma$-algebra of $\Omega$, and $\mathbb{P}$ is the associated probability measure. Our focus is on computing statistical moments in the scattering cross-section of the obstacle when key properties such as the obstacle's location or orientation are random.

The scattering cross-section is a function of the direction from which the incident sound originates. We assume an incident plane wave $\boldsymbol{u}^{\text {inc }}(\boldsymbol{x} ; \widehat{\mathbf{d}})=$ $e^{i k x \cdot \widehat{d}}$ that strikes the configuration from the direction $-\widehat{\mathbf{d}}$ with wavelength $\lambda=$ $2 \pi / k$, where $k$ is the wavenumber. The configuration is subjected to incident waves from various directions. For each such direction $\widehat{\mathbf{d}}$, the incident wave induces a scattered field $u(x, \omega ; \widehat{d})$ that satisfies the Helmholtz equation,

$$
\triangle \mathfrak{u}(\boldsymbol{x}, \omega ; \widehat{\mathbf{d}})+k^{2} u(x, \omega ; \widehat{d})=0, \quad x \in \mathbb{R}^{2} \backslash \overline{\mathrm{D}(\omega)},
$$

the radiation condition,

$$
\lim _{|x| \rightarrow \infty} \sqrt{|x|}\left[\frac{\partial u(x, \omega ; \widehat{d})}{\partial x}-i k u(x, \omega ; \widehat{d})\right]=0,
$$

uniformly in all directions $\widehat{\boldsymbol{x}}=\boldsymbol{x} /|\boldsymbol{x}|$, and the sound soft boundary condition,

$$
u(x, \omega ; \widehat{d})=-u^{\text {inc }}(x ; \widehat{d}), \quad x \in \partial D(\omega),
$$

where $\partial \mathrm{D}(\omega)$ denotes the random boundary of $D(\omega)$. Thus, for a given incident direction $\widehat{\mathbf{d}}$, the scattered field $\boldsymbol{u}(\boldsymbol{x}, \boldsymbol{\omega} ; \widehat{\mathbf{d}})$ is a random process whose 
behaviour a long way from a scatterer is described by the far field,

$$
u^{\infty}(\widehat{x}, \omega ; \widehat{d})=\lim _{|x| \rightarrow \infty} \sqrt{|x|} e^{-i k|x|} \mathfrak{u}(x, \omega ; \widehat{d}), \quad \widehat{x}=\frac{x}{|x|} .
$$

In several applications, including climate science, an important quantity associated with the scattered field is its intensity, which at large distances $|\boldsymbol{x}|$ from the scatterer is $[8$, p. 29]

$$
I(x ; \widehat{d})=\frac{\left|u^{\infty}(\widehat{x}, \omega ; \widehat{d})\right|^{2}}{|x|}, \quad \widehat{x}=\frac{x}{|x|} .
$$

Integrating the intensity over the unit circle $S$ yields the scattering crosssection [8, p. 12] for the incident direction $\widehat{\mathbf{d}}$,

$$
C_{\text {sca }}(\omega ; \widehat{d})=\int_{S}\left|\mathfrak{u}^{\infty}(\widehat{x}, \omega ; \widehat{d})\right|^{2} \operatorname{ds}(\widehat{x}) .
$$

The optical scattering cross-section of ice and dust particles (which typically have rough surfaces) is an important parameter in climate models. Here roughness corresponds to various bumpiness in the boundary of the particles and does not relate to non-differentiability of the boundary parameterisation maps. For a class of particles in climate applications, the boundary of the particles are described through appropriately truncated Fourier series expansions [2, and references therein].

Our goal is to find, for each incident direction $\widehat{\mathbf{d}} \in \mathrm{S}$, the average value of the scattering cross-section,

$$
\overline{\mathrm{C}_{\mathrm{sca}}}(\widehat{\mathbf{d}})=\mathbb{E}\left[\mathrm{C}_{\mathrm{sca}}(\cdot ; \widehat{\mathbf{d}})\right]=\int_{\Omega} \mathrm{C}_{\mathrm{sca}}(\omega ; \widehat{\mathbf{d}}) \mathrm{d} \mathbb{P}(\omega),
$$

and the variance

$$
\operatorname{var}\left(\mathrm{C}_{\mathrm{sca}}\right)(\widehat{\mathbf{d}})=\mathbb{E}\left[\left(\mathrm{C}_{\mathrm{sca}}(\cdot ; \widehat{\mathbf{d}})-\overline{\mathrm{C}_{\mathrm{sca}}}(\widehat{\mathbf{d}})\right)^{2}\right]
$$


in the case where the stochastic configuration $D(\omega)=D_{1}(\omega) \cup \cdots \cup D_{M}(\omega)$ is an ensemble of $M$ disjoint particles $D_{1}(\omega), \ldots, D_{M}(\omega)$ with fixed shape given by a template $\widehat{D}$, but with random orientation and position. Thus the random features of the configuration $D(\omega)$ are described by the orientations $\phi_{1}(\boldsymbol{z}), \ldots, \phi_{M}(\boldsymbol{z})$ and centres $\boldsymbol{x}_{1}(\boldsymbol{z}), \ldots, \boldsymbol{x}_{M}(\boldsymbol{z})$ of $D_{1}, \ldots, D_{M}$, respectively, where $z(\omega)=\left(z_{1}(\omega), \ldots, z_{N}(\omega)\right)$, and $z_{1}(\omega), \ldots, z_{N}(\omega)$ are independent random variables. For convenience, we assume that the random variables are uniformly distributed. However, for beta or normal or log-normal distributions, only minor changes are required in our algorithm, as explain in Section 3. In particular, the random scatterer $D_{I}(\omega)$ is obtained by rotation of the template scatterer $\widehat{D}$ counterclockwise by an angle $\phi_{I}(\omega)$ and then translation by $\boldsymbol{x}_{\mathrm{I}}(\omega)$ for $\mathrm{I}=1, \ldots, M$.

A standard tool for uncertainty quantification is the Monte Carlo method. A disadvantage of using the Monte Carlo method (to approximate the integrals in the statistical moments computation with $m$ sample points) is its slow $1 / \sqrt{m}$ convergence rate, which necessitates a large number of simulations even for, say, two-digit accuracy. In this work we reduce the number of simulations required by using high order orthogonal polynomial expansions of the scattering crosssection, and further approximate the unknown coefficients in the expansions using high order quadrature sampling. This approach belongs to the class of the stochastic collocation generalised polynomial chaos (gPC) method and is widely used for solving stochastic partial differential equations [7]. As described by Maître and Kino [7], for a large number of random variables, the curse of dimensionality in the stochastic collocation approach can be treated using the adaptive sparse grid technique. Even for relatively small numbers of random variables (as considered in this work), an efficient forward solver plays a crucial role to simulate the associated deterministic system for each sample collocation point.

We show in Section 4 that, even for a single fixed observation direction $\widehat{\mathbf{d}}$, tens or even hundreds of deterministic simulations are required to compute the average scattering cross-section $\overline{\mathrm{C}_{\text {sca }}}(\widehat{\mathbf{d}})$, even using the spectrally accurate stochastic collocation method with a few random variables. Our goal is to 
compute $\overline{\mathrm{C}_{\text {sca }}}(\widehat{\mathbf{d}})$ as a function of the observation direction $\widehat{\mathbf{d}}$ and visualize the mean and scattering cross-section across all directions covering the configurations. In order to plot at one thousand incident directions, say, we need to perform hundreds of thousands of simulations. Thus it is essential to use an efficient deterministic scattering algorithm for the simulations.

In a recent work [6], we described a variant of the stochastic scheme for the bistatic acoustic cross-section at a single incident direction, based on using an iterative solver with fast multiplication using the T-matrix of each scatterer in the configuration, and the translation addition theorem. However, for the scattering cross-section problem considered in this work, for which we need to consider hundreds of thousands of incident directions, the number of simulations is so large that it is not feasible to use an iterative solver. In contrast, in this case it is efficient to use an integral equation based forward solver, compared to the PDE based solver used previously [6].

Motivated by the large number of incident directions, we use a surface integral representation for the scattered field. This representation readily facilitates computation of the far field required in (6) to compute the scattering crosssection. We solve the resulting surface integral equation using an efficient spectral algorithm $[3,4,5]$. The high order convergence of the spectral forward solver means that the resulting linear system has relatively few unknowns per scatterer, so that for the small numbers of scatterers considered in this work the discretisation matrix can be assembled in memory and its LU factorisation computed and stored. Once the LU factorisation is computed, we can solve for even thousands of incident directions very quickly. Thus, by using the spectral algorithm, we substantially reduce the computation complexity impact of the large number of incident directions in the algorithm.

In Section 2 we describe our efficient algorithm for deterministic multiple scattering simulations. In Section 3 we describe our gPC stochastic collocation scheme. Finally, in Section 4 we demonstrate the efficiency and accuracy of our combined gPC and spectrally accurate boundary integral equation algorithm with numerical results for a configuration of rough scatterers. 


\section{Deterministic multiple scattering simulations}

In this section we describe our efficient algorithm for simulating the scattering cross-section $\mathrm{C}_{\mathrm{sca}}(\boldsymbol{z} ; \widehat{\mathbf{d}})$ induced by the incident plane wave with direction $\widehat{\mathbf{d}}$, where $\boldsymbol{z}$ is a sample of the random vector $\boldsymbol{z}(\boldsymbol{\omega})$. We consider the location and orientation of the $M$ closed and bounded scatterers $D_{1}, \ldots, D_{M}$ to be fixed. Without loss of generality, for each $J=1, \ldots, M$, we assume that the closed boundary curve $\partial \mathrm{D}_{\mathrm{J}}$ is parametrised by a $2 \pi$-periodic map.

Due to the unbounded multiply-connected domain $\mathbb{R}^{2} \backslash \overline{\mathrm{D}(\boldsymbol{z})}$ and our interest in the far field (to compute the scattering cross-section), it is natural to first start with a boundary integral representation of the scattered field:

$$
\mathfrak{u}(\boldsymbol{x}, \boldsymbol{z} ; \widehat{\mathbf{d}})=\sum_{J=1}^{M} \int_{\partial D_{\mathrm{J}}(z)}\left[\frac{\partial \Phi(\boldsymbol{x}, \mathbf{y})}{\partial \mathfrak{n}(\mathbf{y})}-\mathfrak{i} \eta \Phi(\boldsymbol{x}, \mathbf{y})\right] \psi_{\mathrm{J}}(\mathbf{y}, \boldsymbol{z} ; \widehat{\mathbf{d}}) \mathrm{ds}(\mathbf{y})
$$

for $\boldsymbol{x} \in \mathbb{R}^{2} \backslash \overline{\mathrm{D}(\boldsymbol{z})}$. Here, $\mathbf{n}(\mathbf{y})$ is the unit outward normal to the boundary $\partial \mathrm{D}_{\mathrm{J}}(\boldsymbol{z})$ of $\mathrm{D}_{\mathrm{J}}(\boldsymbol{z})$ at $\boldsymbol{y}$ and $\Phi(\boldsymbol{x}, \boldsymbol{y})=\frac{i}{4} \mathrm{H}_{0}^{(1)}(k|\boldsymbol{x}-\boldsymbol{y}|)$ is the fundamental solution of the two dimensional Helmholtz equation and $\mathrm{H}_{0}^{(1)}$ is the Hankel function of the first kind. Using the boundary condition (3) and the jump relations for the double and single layer potential [3], we obtain the system of integral equations for the unknown densities $\psi_{1}, \ldots, \psi_{M}$ in (9) on the boundaries of the configuration:

$$
\psi_{\mathrm{I}}(\boldsymbol{x}, \boldsymbol{z} ; \widehat{\mathbf{d}})+\sum_{\mathrm{J}=1}^{\mathrm{M}}\left(\mathcal{K}_{\mathrm{IJ}} \psi_{\mathrm{J}}-i \eta \mathcal{S}_{\mathrm{IJ}} \psi_{\mathrm{J}}\right)(\boldsymbol{x}, \boldsymbol{z} ; \widehat{\mathbf{d}})=-2 \mathrm{u}^{\mathrm{inc}}(\boldsymbol{x} ; \widehat{\mathbf{d}})
$$

for $\boldsymbol{x} \in \partial \mathrm{D}_{\mathrm{I}}(\boldsymbol{z})$ and $\mathrm{I}=1, \ldots, M$. This equation has a unique solution for all wavenumbers provided the coupling parameter $\eta$ has nonzero real part. The double and single layer operators $\mathcal{K}_{\mathrm{IJ}}$ and $\mathcal{S}_{\mathrm{IJ}}$, with source density defined 
on $\partial \mathrm{D}_{\mathrm{J}}(\boldsymbol{z})$ and the acoustic-potential fields observed at $\boldsymbol{x} \in \partial \mathrm{D}_{\mathrm{I}}(\boldsymbol{z})$, are

$$
\mathcal{K}_{\mathrm{IJ}} v(\boldsymbol{x})=2 \int_{\partial \mathrm{D}_{\mathrm{J}}(\boldsymbol{z})} \frac{\partial \Phi(\boldsymbol{x}, \mathbf{y})}{\partial \mathbf{n}(\mathbf{y})} v(\mathbf{y}) \mathrm{ds}(\mathbf{y}), \quad \boldsymbol{x} \in \partial \mathrm{D}_{\mathrm{I}}(\boldsymbol{z})
$$

and

$$
\left(\mathcal{S}_{\mathrm{IJ}} v\right)(\boldsymbol{x})=2 \int_{\partial \mathrm{D}_{\mathrm{J}}(\boldsymbol{z})} \Phi(\boldsymbol{x}, \boldsymbol{y}) v(\mathbf{y}) \mathrm{ds}(\mathbf{y}), \quad \boldsymbol{x} \in \partial \mathrm{D}_{\mathrm{I}}(\boldsymbol{z}) .
$$

Once the surface densities $\psi_{1}, \ldots, \psi_{M}$ have been computed, the far field is [3, equation (3.64)]

$$
u^{\infty}(\widehat{x}, z ; \widehat{\mathbf{d}})=\frac{1+i}{4 \sqrt{\pi k}} \sum_{J=1}^{M} \int_{\partial D_{J}(z)}\left[\frac{\partial e^{-i k \widehat{x} \cdot y}}{\partial \mathfrak{n}(\mathbf{y})}-i \eta e^{-i k \widehat{x} \cdot y}\right] \phi_{J}(\mathbf{y}, z ; \widehat{\mathbf{d}}) \mathrm{ds}(\mathbf{y})
$$

To numerically evaluate the integral on the unit circle in (6) we use the rectangle rule, with quadrature points $\widehat{x}_{j}$ and weights $\mu_{j}$,

$$
\widehat{x}_{j}=\left(\cos \phi_{j}, \sin \phi_{j}\right), \quad \phi_{j}=\frac{2 \pi j}{m-1}, \quad \mu_{j}=\frac{2 \pi}{m}, \quad j=0, \ldots, m-1 .
$$

Due to the integration on the periodic domain, this rule has high order accuracy for smooth integrands. Thus our approximation to the scattering cross-section of the configuration is

$$
\mathrm{C}_{\mathrm{sca}}(\boldsymbol{z} ; \widehat{\mathbf{d}})=\sum_{j=0}^{\mathrm{m}-1} \mu_{j}\left|\mathrm{u}^{\infty}\left(\widehat{\boldsymbol{x}}_{j}, \boldsymbol{z} ; \widehat{\mathbf{d}}\right)\right|^{2}
$$

Next we focus on efficient computation of the unknown densities in (13) that requires efficient discretisation of the $M$-coupled surface boundary integral equations (10).

The first ingredient in our efficient scheme for simulation of the scattering cross-section is the parametrisation of each boundary $\partial \mathrm{D}_{\mathrm{J}}(\boldsymbol{z})$ using a mapping

$$
\mathbf{q}_{\mathrm{J}}(\boldsymbol{z}): S \rightarrow \partial \mathrm{D}_{\mathrm{J}}(\boldsymbol{z}) \text {. }
$$


This parametrisation facilitates the use of a pseudospectral Galerkin discretisation of the integral equation (10) using the trial space of trigonometric polynomials of degree, say, at most L'. High order representation of the surface densities and corresponding high order approximation of the transplanted integral operators on the unit circle $S$ means that relatively few unknowns are needed in the discretisation to obtain a specific accuracy in the solution.

Discretisation of the system (10) leads to the block linear system

$$
\boldsymbol{v}_{\mathrm{I}}+\sum_{\mathrm{J}=1}^{\mathrm{M}}\left(\mathrm{K}_{\mathrm{IJ}}-\mathrm{i \eta} \mathrm{S}_{\mathrm{IJ}}\right) \boldsymbol{v}_{\mathrm{J}}=\mathbf{b}_{\mathrm{I}}(\widehat{\mathbf{d}}), \quad \text { for } \mathrm{I}=1, \ldots, \mathrm{M},
$$

where $\mathbf{b}_{\mathrm{I}}(\widehat{\mathbf{d}})$ is the discretisation of $\left.\boldsymbol{u}^{\mathrm{inc}}(\cdot, \boldsymbol{z} ; \widehat{\mathbf{d}})\right|_{\partial \mathrm{D}_{\mathrm{I}}(\boldsymbol{z})}$, and $\mathrm{K}_{\mathrm{IJ}}, \mathrm{S}_{\mathrm{IJ}}$ are spectrally accurate discretisations of $\mathcal{K}_{\mathrm{IJ}}$ and $\mathcal{S}_{\mathrm{IJ}}$, respectively. Crucially, the matrix in the left hand side of (15) is independent of the incident direction $\widehat{\mathbf{d}}$. Thus, to represent $C_{\text {sca }}(\boldsymbol{z} ; \widehat{\mathbf{d}})$ for many incident directions $\widehat{\mathbf{d}}=\widehat{\mathbf{d}}_{1}, \ldots, \widehat{\mathbf{d}}_{\mathrm{K}}$, we need to solve many linear systems with the same matrix but many right hand sides derived from $\widehat{\mathbf{d}}_{1}, \ldots, \widehat{\mathbf{d}}_{\mathrm{k}}$. Thus the second step in our efficient simulation algorithm is to assemble the matrix and compute its LU factorisation. Using the LU factorisation these linear systems are solved very efficiently in almost the same CPU time as the simulation for a single incident direction. Three dimensional counterparts of such efficient discretisations are described in our earlier work $[4,5]$.

\section{Uncertainty quantification}

In this section we describe our high order generalised polynomial chaos method for uncertainty quantification of the stochastic cross-section. We first describe the procedure in the case that our scattering configuration is described by a single uniformly distributed random variable $z$ with mean zero and variance $\sigma^{2}$, that is $z \sim \mathcal{U}(-\sqrt{3} \sigma, \sqrt{3} \sigma)$ with probability density function $\rho(z)=\frac{1}{2 \sqrt{3} \sigma} \chi_{(-\sqrt{3} \sigma, \sqrt{3} \sigma)}(z)$ and associated probability measure $\mathbb{P}$. In 
case of beta or normal (or log-normal) distributions, the Legendre polynomial based details below are replaced with similar derivations using Jacobi or Hermite polynomials, respectively [7].

Let $P_{n}$ denote the Legendre polynomial of degree $n$ that satisfies the recurrence relation [1, equation (22.7.10)],

$$
(n+1) P_{n+1}(x)=(2 n+1) x P_{n}(x)-n P_{n-1}(x),
$$

for real $x$, with $P_{0}(x)=0, P_{1}(x)=x$. The Legendre polynomials are orthogonal with respect to the standard inner product on the interval $[-1,1]$, satisfying [1, equation (22.2.10)]

$$
\frac{2 n+1}{2} \int_{-1}^{1} P_{n}(x) P_{m}(x) d x=\delta_{m, n} .
$$

Using a change of variables $z=\sqrt{3} \sigma x$ we define

$$
\widehat{P}_{n}(z)=P_{n}\left(\frac{z}{\sqrt{3} \sigma}\right)=P_{n}(x) .
$$

Therefore,

$$
\mathbb{E}\left[\widehat{P}_{\mathfrak{n}}(\cdot) \widehat{P}_{\mathfrak{m}}(\cdot)\right]:=\int_{-\sqrt{3} \sigma}^{\sqrt{3} \sigma} \widehat{P}_{\mathfrak{n}}(z) \widehat{P}_{\mathfrak{m}}(z) d \mathbb{P}(z)=\frac{1}{2 \mathfrak{n}+1} \delta_{\mathfrak{m}, \mathfrak{n}} .
$$

Thus the polynomials $\widehat{P}_{n}$ are orthogonal with respect to the Radon-Nikodym derivative $d \mathbb{P}(z)=\rho(z) d z$. Equation (19) motivates the inner product for square integrable functions $f, g$ with respect to the measure $d \mathbb{P}(z)$,

$$
\begin{aligned}
\langle f, g\rangle & =\mathbb{E}[f(\cdot) g(\cdot)]=\int_{-\sqrt{3} \sigma}^{\sqrt{3} \sigma} f(z) \overline{g(z)} \frac{1}{2 \sqrt{3} \sigma} d z \\
& =\int_{-1}^{1} f(\sqrt{3} \sigma x) g(\sqrt{3} \sigma x) \frac{1}{2} d x .
\end{aligned}
$$


In practice we need the discrete approximation to the inner product (20),

$$
\langle f, g\rangle_{L}=\sum_{m=1}^{L+1} v_{m} f\left(\sqrt{3} \sigma x_{m}\right) \overline{g\left(\sqrt{3} \sigma x_{m}\right)},
$$

obtained by evaluation of the integral in (20) using the Gauss-Legendre rule [1, equation (25.4.29)]. The knots $x_{m}$ for $m=1, \ldots, L+1$ are the zeros of $\mathrm{P}_{\mathrm{L}+1}$, whilst the weights (incorporating the $1 / 2$ factor due to the probability measure on $[-1,1])$ are

$$
v_{m}=\frac{1}{(L+1)\left[P_{L}\left(x_{m}\right)-x_{m} P_{L+1}\left(x_{m}\right)\right]}, \quad m=1, \ldots, L+1 .
$$

We are now able to compute the discrete orthogonal projection of the scattering coefficient $C_{\text {sca }}(z ; \widehat{\mathbf{d}})$ onto the space $V_{L}=\operatorname{span}\left\{\widehat{P}_{0}, \ldots, \widehat{P}_{L}\right\}$, for some $L \in \mathbb{N}$, using the discrete inner product (21) on $\mathrm{V}_{\mathrm{L}}$. In particular,

$$
\mathrm{C}_{\mathrm{sca}}(z ; \widehat{\mathbf{d}}) \approx \sum_{\mathrm{l}=0}^{\mathrm{L}} \widehat{\mathrm{C}_{\mathrm{sca}, \mathrm{l}}}(\widehat{\mathbf{d}}) \mathrm{P}_{\mathrm{l}}(z)
$$

where, using (18), (19) and (21),

$$
\begin{aligned}
\widehat{\mathrm{C}_{\mathrm{sca}, l}}(\widehat{\mathbf{d}}) & =(2 l+1)\left\langle\mathrm{C}_{\mathrm{sca}}(\cdot ; \widehat{\mathbf{d}}), \widehat{\mathrm{P}}_{\mathrm{l}}(\cdot)\right\rangle_{\mathrm{L}} \\
& =(2 l+1) \sum_{\mathrm{m}=1}^{\mathrm{L}+1} v_{\mathrm{m}} \mathrm{C}_{\mathrm{sca}}\left(\sqrt{3} \sigma x_{\mathrm{m}} ; \widehat{\mathbf{d}}\right) \mathrm{P}_{\mathrm{l}}\left(x_{\mathrm{m}}\right) .
\end{aligned}
$$

As described in Section 1, we parametrise the stochastic features in our model with $M$ particles by the random vector $z=\left(z_{1}, \ldots, z_{N}\right) \in \mathbb{R}^{N}$, with associated measure $\mathbb{P}_{N}(\boldsymbol{z})$, where $N \leqslant 3 M$. We assume that the $N$ random variables $z_{1}, \ldots, z_{\mathrm{N}}$ have uniform distribution with mean zero and variances $\sigma_{1}^{2}, \ldots, \sigma_{\mathrm{N}}^{2}$, respectively. Analogously to the univariate case above, we introduce the tensor product polynomial

$$
\widehat{\mathcal{P}}_{l}(z)=\widehat{\mathrm{P}}_{\mathrm{l}_{1}}\left(z_{1}\right) \cdots \widehat{\mathrm{P}}_{\mathrm{l}_{\mathrm{N}}}\left(z_{\mathrm{N}}\right),
$$


where $\mathbf{l}=\left(l_{1}, \ldots, l_{N}\right)$. Using a similar change of variables to above, we obtain the tensor product analogue of (18),

$$
\widehat{\mathcal{P}}_{\mathbf{l}}(z)=\mathcal{P}_{l}(x)=\mathrm{P}_{\mathrm{l}_{1}}\left(x_{1}\right) \cdots \mathrm{P}_{\mathrm{l}_{\mathrm{N}}}\left(\mathrm{x}_{\mathrm{N}}\right),
$$

where $\boldsymbol{x}=\left(x_{1}, \ldots, x_{N}\right)$ and $z=\left(z_{1}, \ldots, z_{N}\right)=\left(\sqrt{3} \sigma_{1} x_{1}, \ldots, \sqrt{3} \sigma_{N} x_{N}\right)$. The polynomials $\widehat{\mathcal{P}}_{1}$ are easily seen to be orthogonal with respect to the measure $\mathbb{P}_{\mathrm{N}}(\boldsymbol{z})$. Indeed, with respect to the tensor product analogue of the inner product in (20),

$$
\left\langle\widehat{\mathcal{P}}_{\mathfrak{n}}(\cdot), \widehat{\mathcal{P}}_{\mathbf{m}}(\cdot)\right\rangle= \begin{cases}\frac{1}{\left(2 \mathfrak{n}_{1}+1\right)} \cdots \frac{1}{\left(2 \mathfrak{n}_{\mathrm{N}}+1\right)}, & \text { when } \mathfrak{n}=\mathbf{m} \\ 0, & \text { otherwise }\end{cases}
$$

Using the change of variables and the tensor product version of the discrete inner product (21), we obtain our spectrally accurate approximation to the scattering cross- section of the stochastic configuration:

$$
\mathrm{C}_{\mathrm{sca}}(\boldsymbol{z} ; \widehat{\mathbf{d}}) \approx \mathrm{C}_{\mathrm{sca}, \mathrm{L}}(\boldsymbol{z} ; \widehat{\mathbf{d}}):=\sum_{\mid \|_{\infty} \leqslant \mathrm{L}} \widehat{\mathrm{C}_{\mathrm{sca}, \mathrm{l}}}(\widehat{\mathbf{d}}) \mathcal{P}_{\mathrm{l}}(\boldsymbol{z})
$$

where $\mid \mathbf{l}_{\infty}=\max \left\{l_{1}, \ldots, l_{N}\right\}$, and

$$
\widehat{\mathrm{C}_{\mathrm{sca}, l}}(\widehat{\mathbf{d}})=\left(2 \mathrm{l}_{1}+1\right) \cdots\left(2 \mathrm{l}_{\mathrm{N}}+1\right) \sum_{\mid \mathrm{l}_{\infty} \leqslant \mathrm{L}+1} v_{\mathrm{m}_{1}} \cdots v_{\mathrm{m}_{\mathrm{N}}} \mathrm{C}_{\mathrm{sca}}\left(z_{\mathrm{m}} ; \widehat{\mathbf{d}}\right) \mathcal{P}_{\mathrm{l}}\left(x_{\mathrm{m}}\right) \text {, }
$$

and $\boldsymbol{z}_{\mathrm{m}}=\left(\sqrt{3} \sigma_{1} x_{\mathrm{m}_{1}}, \ldots, \sqrt{3} \sigma_{\mathrm{N}} x_{\mathrm{m}_{\mathrm{N}}}\right), \boldsymbol{x}_{\mathrm{m}}=\left(x_{\mathrm{m}_{1}}, \ldots, x_{\mathrm{m}_{\mathrm{N}}}\right)$. Using the gPC coefficients in (24) we compute approximations to the mean value and variance as

$$
\overline{\mathrm{C}_{\mathrm{sca}}}(\widehat{\mathbf{d}}) \approx \widehat{\mathrm{C}_{\mathrm{sca}, 0}}(\widehat{\mathbf{d}}), \quad \operatorname{var}\left(\mathrm{C}_{\mathrm{sca}}\right)(\widehat{\mathbf{d}}) \approx \sum_{\mid \mathrm{l}_{\infty}=1}^{\mathrm{L}+1}\left(2 \mathrm{l}_{1}+1\right) \cdots\left(2 \mathrm{l}_{\mathrm{N}}+1\right)\left|\widehat{\mathrm{C}_{\mathrm{sca}, \mathrm{l}}}(\widehat{\mathbf{d}})\right|^{2}
$$

In practice, if the number of random variables $\mathrm{N}$ is greater than or equal to five, then it is important to replace $|\boldsymbol{l}|_{\infty}$ in the above algorithm with $|\boldsymbol{l}|=\sum_{i=1}^{N} l_{i}$ and use a sparse grid version of the quadrature rule to tackle the curse of stochastic dimension [7]. 


\section{Numerical results}

We present numerical results for a stochastic configuration comprising two rough particles (with rough boundary; see Figure 1) that are rotations and translations of a template particle of diameter five wavelengths. The orientations $\phi_{1}$ and $\phi_{2}$ of the two particles are given by independent random variables with uniform distribution $\mathcal{U}(-\sqrt{3} / 10, \sqrt{3} / 10)$ and the $x$-coordinate of the second particle is given by an independent random variable with uniform distribution $\mathfrak{U}(-\sqrt{3} / 20, \sqrt{3} / 20)$. The first particle is fixed and so the random $x$-coordinate of the second scatterer determines the separation of the two particles. Thus the random parameter vector describing the configuration is in the probability space $\left(\mathbb{R}^{3}, \mathcal{F}_{3}, \mathbb{P}_{3}\right)$. The computed scattering cross-section is invariant under fixed shifts of the configuration and hence we are effectively quantifying the uncertainty in the scattering cross-section with respect to the random orientation and separation of the particles.

In Figure 1 we illustrate the mean configuration, in which the first particle has centre $(-2,0)$ and the second particle has centre $(2,0)$, and visualise the total field $\mathfrak{u}(\boldsymbol{x}, \boldsymbol{\omega} ; \widehat{\mathbf{d}})+\mathfrak{u}^{\text {inc }}(\boldsymbol{x} ; \widehat{\mathbf{d}})$ induced by scattering of an incident plane wave with direction $\widehat{\mathbf{d}}=(0,1)$.

We used our efficient hybrid gPC and spectral integral equation algorithm to compute the approximate mean scattering cross-section of the random configuration and its variance for maximum polynomial degree L between 5 and 15. In Figure 2 we visualise the approximate mean scattering crosssection, computed with maximum polynomial degree $L=5$, as a function of incident direction $\widehat{\mathbf{d}}(\theta)=(\cos \theta, \sin \theta)$ in polar coordinates. The uncertainty is visualised by shading the region between the curves

$$
\overline{\mathrm{C}_{\mathrm{sca}, \mathrm{L}}}(\widehat{\mathbf{d}})-\sqrt{\operatorname{var}\left(\mathrm{C}_{\mathrm{sca}, \mathrm{L}}\right)(\widehat{\mathbf{d}})}, \quad \overline{\mathrm{C}_{\mathrm{sca}, \mathrm{L}}}(\widehat{\mathbf{d}})+\sqrt{\operatorname{var}\left(\mathrm{C}_{\mathrm{sca}, \mathrm{L}}\right)(\widehat{\mathbf{d}})} .
$$

For this problem, the exact value of the mean scattering cross-section is not known. Hence, following standard practice, we use our gPC approximation 


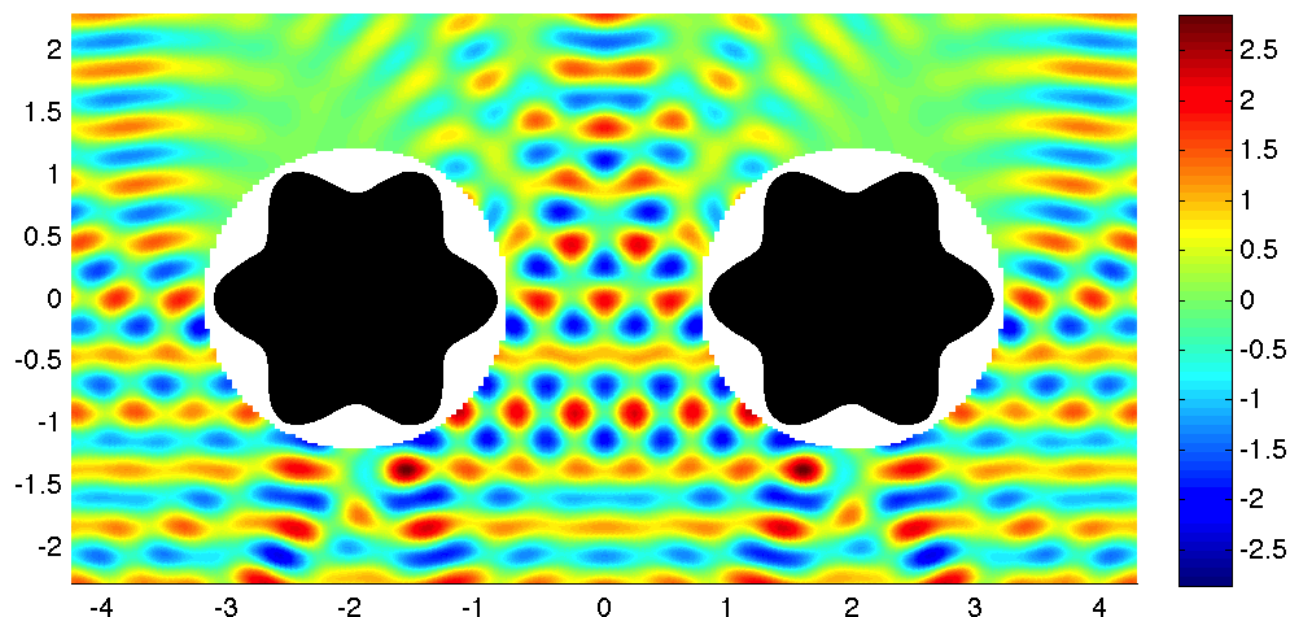

Figure 1: Visualisation of the mean configuration of the scatterers and the total field induced by an incident plane wave with direction $(0,1)$.

obtained with Legendre polynomial degree 15 as a reference solution and approximate the error using gPC polynomials of degree L by

$$
\operatorname{error}_{\mathrm{L}}=\left\|\overline{\mathrm{C}_{\mathrm{sca}, \mathrm{L}}}(\widehat{\mathbf{d}})-\overline{\mathrm{C}_{\mathrm{sca}, 15}}(\widehat{\mathbf{d}})\right\|_{\infty} .
$$

In practice we approximate the maximum norm error by sampling at one thousand equally spaced points on the unit circle. Because we have three random variables, we require $(\mathrm{L}+1)^{3}$ simulations to compute our gPC approximation with polynomials of maximum degree L. In Figure 3 we plot the error against the number of simulations for gPC and Monte Carlo. For this experiment the gPC method with $L=5$ (and hence 216 simulations) gives smaller error than the Monte Carlo method even with 50160 simulations. Finally, in Table 1 we show the efficiency of our spectrally accurate (in space and in random variable) method compared with the Monte Carlo method by presenting CPU times required for the uncertainty quantification using a compute node with two hexacore $2.93 \mathrm{GHz}$ Intel Xeon X5670 processors. 


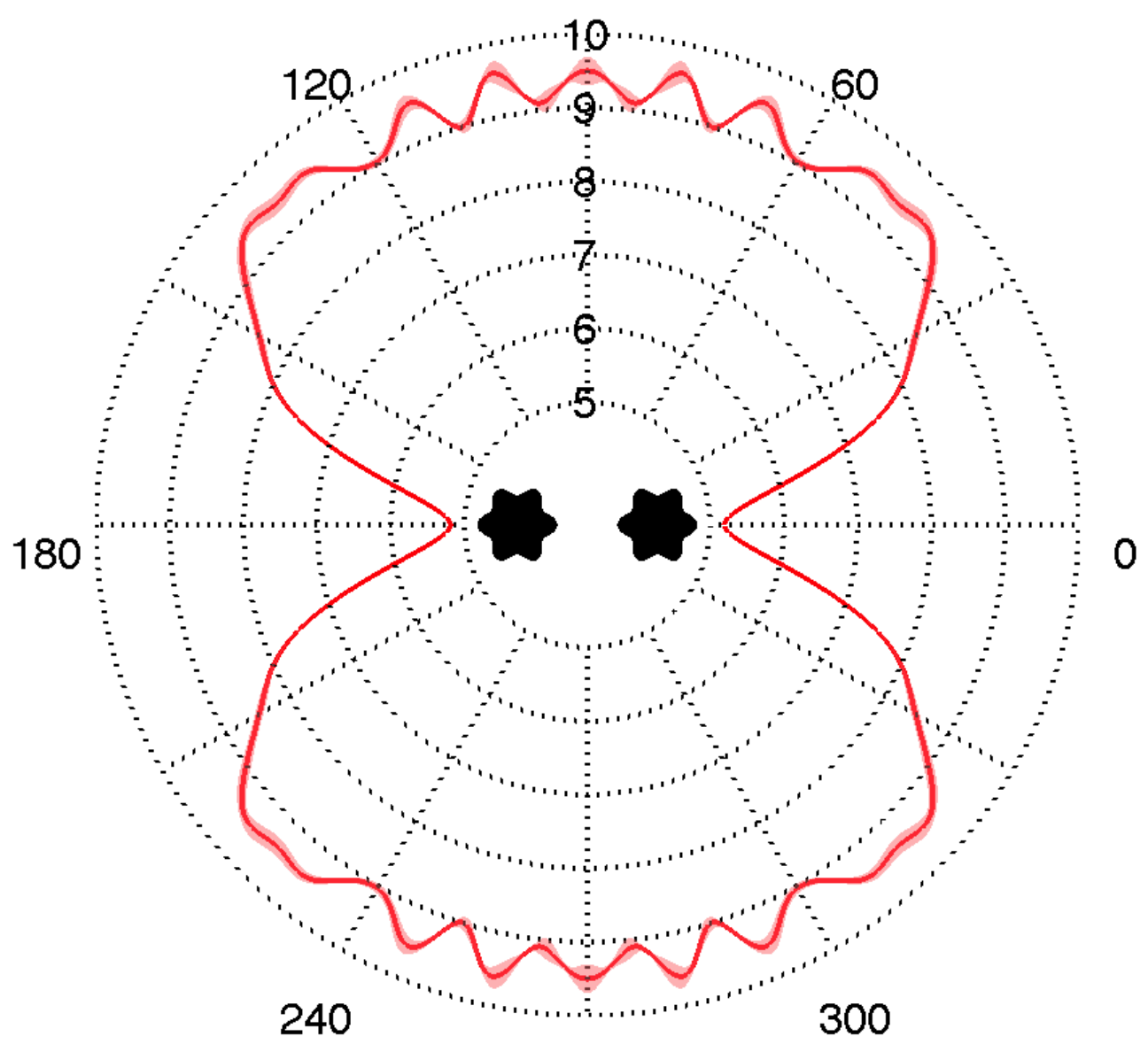

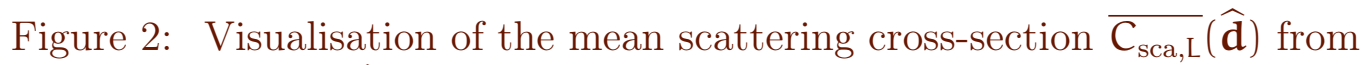
various directions $\widehat{\mathbf{d}}$, of the random configuration (solid line) computed using gPC with $\mathrm{L}=5$ with uncertainty indicated by the shaded region that marks \pm one standard deviation. 
Table 1: CPU time for parallel computation using twelve cores.

\begin{tabular}{cccc} 
Method & Number of simulations & Error & CPU time \\
\hline gPC $(\mathrm{L}=5)$ & 216 & $5.4 \times 10^{-5}$ & $5.1 \mathrm{~min}$ \\
Monte Carlo & 50160 & $1.9 \times 10^{-3}$ & 19.1 hour
\end{tabular}

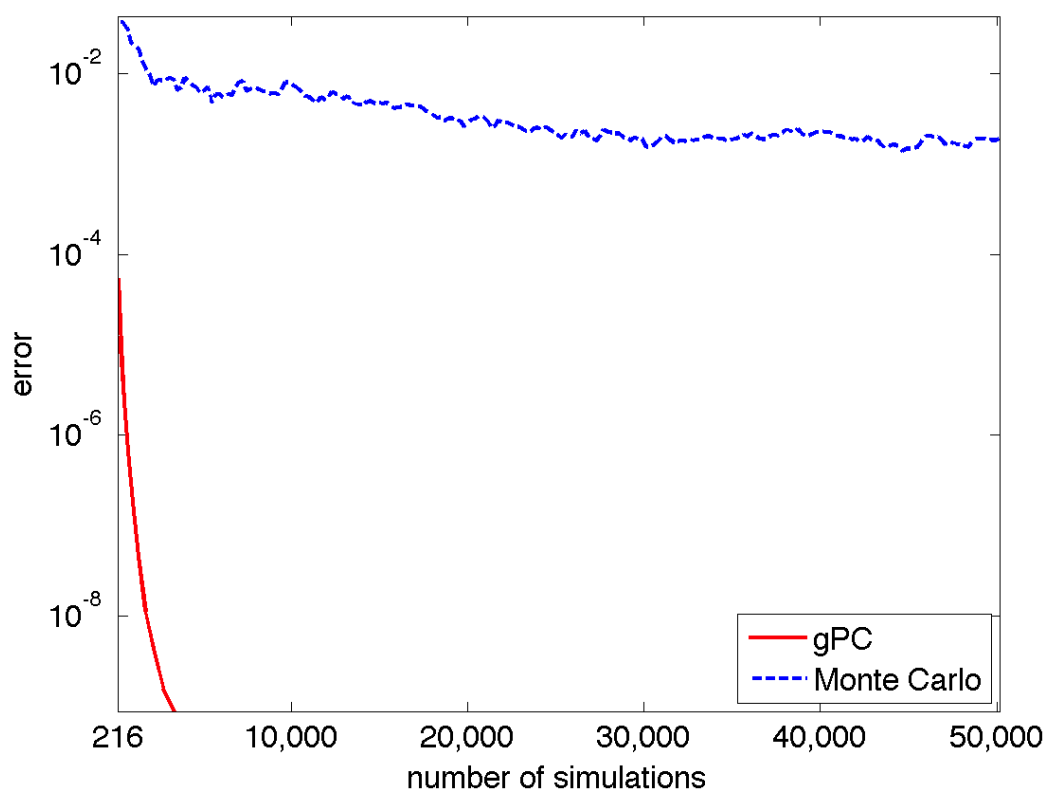

Figure 3: Error in the mean scattering cross-section $\overline{\mathrm{C}_{\text {sca, } \mathrm{L}}}(\widehat{\mathbf{d}})$ computed using gPC with $(L+1)^{3}$ simulations the Monte Carlo method. 
Acknowledgments The research of the first author was supported, in part, by grant DMS-1216889 from the National Science Foundation. Support of the Colorado Golden Energy Computing Organization is gratefully acknowledged.

\section{References}

[1] M. Abramowitz and I. Stegun. Handbook of Mathematical Functions. Dover Publications, New York, 1970. C128, C129

[2] A. Baren. From the single-scattering properties of ice crystals to climate prediction: A way forward. Atmospheric Research, 112:45-69, 2012. doi:10.1016/j.atmosres.2012.04.010. C120, C122

[3] D. Colton and R. Kress. Inverse Acoustic and Electromagnetic Scattering Theory. Springer, 1998. C124, C125, C126

[4] M. Ganesh and I. G. Graham. A high-order algorithm for obstacle scattering in three dimensions. J. Comput. Phys., 198:211-242, 2004. doi:10.1016/j.jcp.2004.01.007. C124, C127

[5] M. Ganesh and S. C. Hawkins. A high-order algorithm for multiple electromagnetic scattering in three dimensions. Numer. Algorithms, 50:469-510, 2009. doi:10.1007/s11075-008-9238-z. C124, C127

[6] M. Ganesh and S. C. Hawkins. A stochastic pseudospectral and T-matrix algorithm for acoustic scattering by a class of multiple particle configurations. J. Quant. Spectrosc. Radiat. Transfer, 2013 (in press). doi:10.1016/j.jqsrt.2013.01.011. C124

[7] O. P. Le Maître and O. M. Kino. Spectral Methods for Uncertainty Quantification. Springer, 2010. C123, C128, C130

[8] H. C. van de Hulst. Light Scattering by Small Particles. Dover, 1957. C120, C122 


\section{Author addresses}

1. M. Ganesh, Department of Applied Mathemeatics and Statistics, Colorado School of Mines, Golden, CO 80401, USA.

mailto:mganesh@mines.edu

2. S. C. Hawkins, Department of Mathematics, Macquarie University, Sydney, NSW 2109, Australia.

mailto:stuart.hawkins@mq.edu.au 